\title{
ORIGINAL
}

\section{Clinician Referrals for Non-opioid Pain Care Following Discontinuation of Long-term Opioid Therapy Differ Based on Reasons for Discontinuation}

\author{
Travis I. Lovejoy, PhD, MPH ${ }^{1,2,3}$, Benjamin J. Morasco, PhD ${ }^{1,2}$, Michael I. Demidenko, BS ${ }^{7}$,
} Thomas H. A. Meath, MPH ${ }^{1,4}$, and Steven K. Dobscha, MD ${ }^{1,2}$

${ }^{1}$ Center to Improve Veteran Involvement in Care,, VA Portland Health Care System, Portland, OR, USA; ${ }^{2}$ Department of Psychiatry, Oregon Health and Science University, Portland, OR, USA; ${ }^{3}$ School of Public Health, Oregon Health and Science University, Portland, OR, USA; ${ }^{4}$ Center for Health Systems Effectiveness, Oregon Health and Science University, Portland, OR, USA.

BACKGROUND: Little is known about pain care offered to patients discontinued from long-term opioid therapy (LTOT) by their prescriber due to aberrant behaviors versus other reasons.

OBJECTIVE: This study aimed to compare rates of nonopioid analgesic pharmacotherapy initiation and clinician referrals for non-pharmacologic pain treatment, complementary and integrative pain therapies, and specialty mental health and substance use disorder treatment between patients discontinued from opioid therapy due to aberrant behaviors versus other reasons.

DESIGN: The design included retrospective manual electronic health record review and administrative data abstraction.

PARTICIPANTS: Patients were sampled from a national cohort of US Department of Veterans Affairs patients prescribed continuous opioid therapy in 2011 who subsequently discontinued opioid therapy in 2012. The study sample comprised 509 patients discontinued from LTOT by opioid-prescribing clinicians.

MAIN MEASURES: The primary independent variable was reason for discontinuation of LTOT (aberrant behaviors versus other reasons). Pain care dichotomous outcomes included clinician use of an opioid taper; initiating new non-opioid analgesic pharmacotherapy; and referrals for non-pharmacologic pain treatment, complementary and integrative pain therapies, and specialty mental health and substance use disorder treatment.

KEY RESULTS: We observed low rates of opioid taper (15\% of patients), initiations of new or modifications of existing non-opioid analgesic pharmacotherapy (45\% of patients), and clinician referrals for non-pharmacologic pain treatment (58\% of patients) and complementary and integrative therapies (25\% of patients). Patients discontinued due to aberrant behaviors, relative to patients discontinued for other reasons, were more likely to receive opioid tapers (adjusted $\mathrm{OR}=5.60,95 \% \mathrm{CI}=2.10$ 14.93), receive new non-opioid analgesic medications or dose changes to an existing non-opioid analgesic medications (adjusted $\mathrm{OR}=2.61,95 \% \mathrm{CI}=1.59-4.29$ ), or be referred for specialty substance use disorder treatment (adjusted OR=7.39, 95\% CI = 3.76-14.53).

CONCLUSIONS: These findings highlight the variability in referral rates for different types of non-opioid pain treatments and challenges accessing specific types of pain care.

Published online April 9, 2018
J Gen Intern Med 33(Suppl 1):S24-S30

DOI: $10.1007 / \mathrm{s} 11606-018-4329-6$

(c) Society of General Internal Medicine (outside the USA) 2018

\section{INTRODUCTION}

US epidemiologic data show rising trends in opioid prescribing nationally from the early 1990 s through 2012 with modest year-over-year declines from 2013 through 2016. ${ }^{1}$ Similar trends have been observed for patients receiving care through the US Department of Veterans Affairs (VA). Nearly 900,000 VA patients were dispensed opioid medication at the prescribing peak in 2012, with rates of opioid prescribing subsequently declining more than $20 \%$ to fewer than 700,000 patients dispensed opioid medication in $2016 .^{2}$ Declining rates of overall opioid prescribing are the result of fewer new opioid initiations as well as discontinuation of existing opioid therapy for long-term users. ${ }^{3}$

Within the VA, the majority of long-term opioid therapy (LTOT) discontinuations among patients with chronic pain are initiated by opioid-prescribing clinicians, rather than patients, and most clinician-initiated discontinuations appear to be due to patient aberrant behaviors such as illicit or non-prescribed controlled substance use, opioid misuse, opioid diversion, and other violations of opioid contracts (e.g., seeking early opioid refills). ${ }^{3}$ Clinical guidelines recommend that clinicians conduct ongoing risk-benefit analyses with patients on LTOT and discontinue opioid therapy when potential harms outweigh benefits of continued treatment. ${ }^{4-5}$ In the context of opioidrelated aberrant behaviors, when opioid therapy presents a risk to individual patients and society, discontinuation is likely the appropriate course of action. Yet clinicians who discontinue patients from LTOT are left with the task of identifying how to best treat these patients' pain without opioids.

Past research has examined non-opioid pain treatment and ancillary care utilization in the context of ongoing opioid therapy. One study compared utilization rates between 5814 VA patients with and without substance use disorders. ${ }^{6} \mathrm{~Pa}-$ tients with substance use disorders, who are at increased risk of being discontinued from LTOT due to aberrant behaviors, ${ }^{3}$ 
had higher pain intensity ratings and rates of non-opioid pain treatment and ancillary pain care utilization, relative to patients without substance use disorders. However, patients with co-occurring substance use disorders are also less responsive to usual pain care and may require more intensive pain treatment using collaborative or other multimodal approaches, ${ }^{7}$ which may explain why pain intensity ratings remain high in patients with substance use disorders despite higher utilization of individual pain treatment modalities. ${ }^{6} \mathrm{Al}-$ though prior studies have characterized pain treatment utilization in the context of ongoing opioid therapy, data are needed about non-opioid pain treatments in patients subsequent to discontinuation from opioid therapy.

The first objective of this study was to identify rates of non-opioid analgesic pharmacotherapy initiation or dose changes to existing medications and clinician referral for non-pharmacologic pain treatment, complementary and integrative pain therapies, and specialty mental health and substance use disorder care in a sample of VA patients whose discontinuation from opioid therapy was initiated by their opioid-prescribing clinicians. A second objective was to compare referral rates between patients discontinued due to aberrant behaviors versus other reasons. We hypothesized that patients discontinued due to aberrant behaviors would be more likely to receive new non-opioid analgesic pharmacotherapy or dose changes to existing medications and have higher referral rates for non-opioid pain treatments and specialty mental health and substance use disorder treatments following LTOT discontinuation.

\section{METHOD}

This study was approved by the VA Portland Health Care System Institutional Review Board.

\section{Sample Selection}

We used the VA Corporate Data Warehouse, a national data repository containing comprehensive information from VA patients' electronic health records, to identify all VA patients prescribed continuous opioid therapy in 2011, excluding patients whose only opioid therapy consisted of agonist treatment for opioid use disorder (i.e., buprenorphine or methadone maintenance). From this cohort, we identified patients who discontinued opioid therapy for at least 12 consecutive months beginning in 2012. We required a minimum of 12 months without opioid prescriptions to ensure that LTOT discontinuation reflected a clinical or personal decision and was not due to circumstances reflecting delays in prescription refills such as extended inpatient stays or geographic relocation. The date of the last opioid prescription fill was designated as the index date for each patient and used as a starting date when initiating the review of patients' electronic health records for opioid discontinuation information. This study focused on LTOT for chronic non-cancer pain, and we thus excluded patients with cancer diagnoses (other than nonmelanoma skin cancer) or surgeries for which opioids may have been prescribed in the year prior to the index date. We also excluded patients with no VA prescriptions or visits and those who died in the 12 months following the index date, as this study aimed to assess pain treatment referrals subsequent to LTOT discontinuation. Additional exclusion criteria included non-veteran status and receiving VA care at non-US hospitals. The final cohort comprised 7247 patients who discontinued LTOT in 2012. In order to identify patients at high risk of discontinuation due to aberrant behaviors for further manual electronic health record review, we randomly sampled 300 patients with substance use disorder diagnoses in the year prior to the index date and propensity score-matched 300 patients without substance use disorder diagnoses. A secondary goal of this study, which has been described in detail elsewhere, ${ }^{3}$ was to compare reasons for discontinuation of LTOT between patients with and without substance use disorders. Propensity score matching allowed us to assemble a sample of patients from this cohort without substance use disorder diagnoses in the year prior to LTOT discontinuation who were otherwise similar to the sample of patients with substance use disorder diagnoses across demographic and clinical variables. Figure 1 details the sample selection for this study.

\section{Data Sources and Study Variables}

Electronic Health Record Review. A trained research associate with experience abstracting data from the VA electronic health record for opioid- and pain-related studies utilized a standardized medical chart abstraction tool to conduct a comprehensive review of patients' VA electronic health records. Additional details about the development of the chart abstraction tool, initial training of the research associate, and ongoing quality assurance monitoring have previously been published. ${ }^{3}$ The electronic health record review identified the date at which the decision was made to discontinue opioid therapy, which may have preceded the date of the last opioid prescription fill (i.e., the index date) when an opioid taper plan was implemented. Electronic health record review identified patient and clinician reasons for discontinuation of opioid therapy. Discontinuation for 509 patients was initiated by the opioid-prescribing clinician, rather than discontinuation being initiated by the patient. These 509 patients formed the analytic sample. Clinician-initiated discontinuation reasons were grouped into two categories: $\left({ }^{1}\right)$ aberrant behaviors, which included aberrant urine drug tests, known or suspected use of alcohol or other substances, opioid misuse, opioid diversion, and non-adherence to the plan of care (e.g., failing to present for a urine drug test when instructed), and $\left(^{2}\right)$ all other reasons, which included patient safety concerns (e.g., patient history of prior opioid overdose, patients with concurrent prescriptions for benzodiazepines), lack of efficacy of opioid therapy (e.g., opioids not reducing pain or improving functioning), no specific reason documented in the medical record, and other reasons, the latter of which comprised $1 \%$ of the analytic sample. 
Patients prescribed opioid therapy for all of 2011, excluding opioid agonist treatment

( methadone maintenance, buprenorphine) $=325,802$

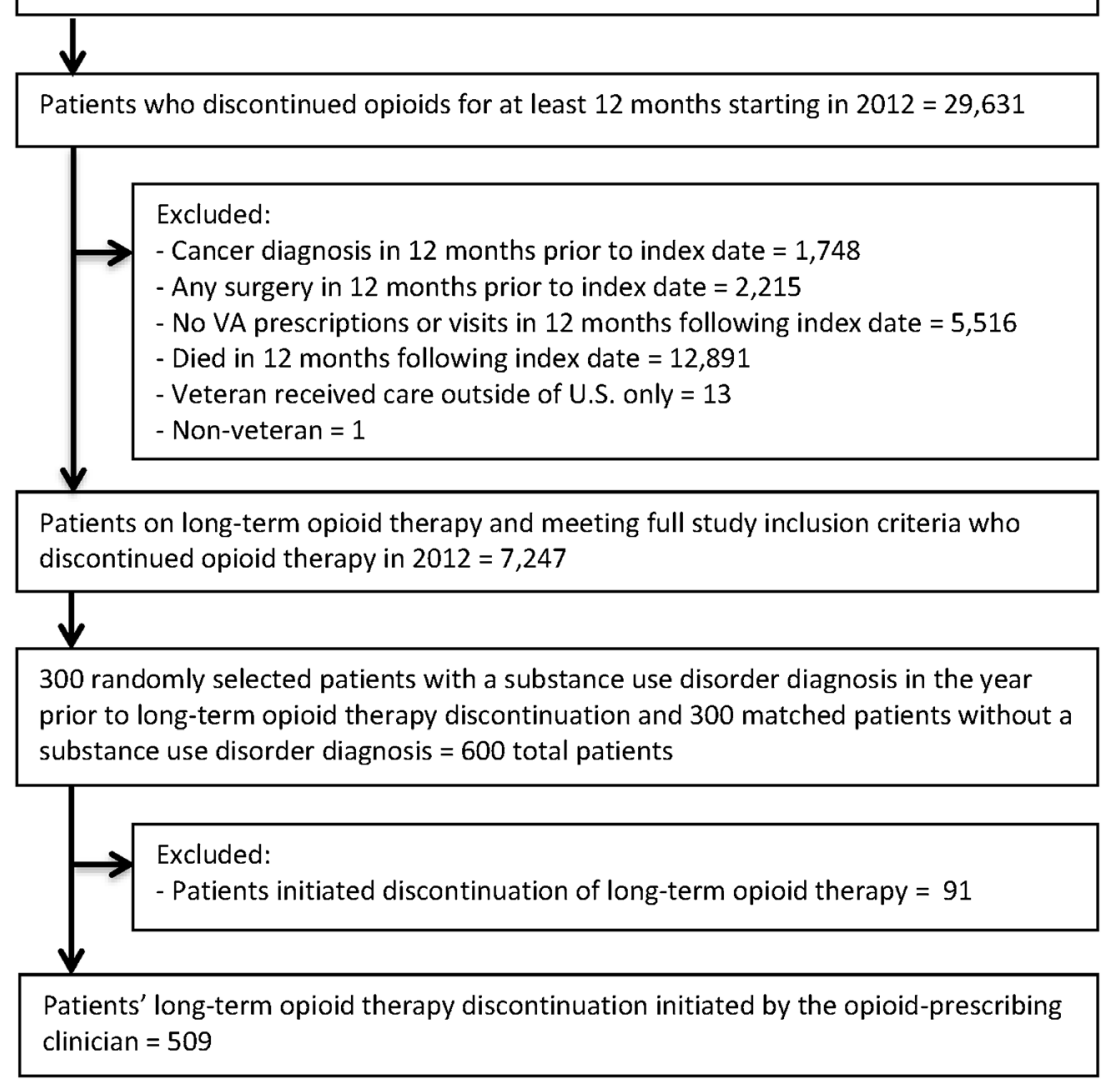

Figure 1 Sample selection for patients who discontinued long-term opioid therapy.

Electronic health record review ascertained the presence of opioid tapers to aid in discontinuation, which we defined as documentation in the medical record of clinicians instructing patients how to gradually reduce daily opioid dose over a defined time interval. Electronic health record review also identified prescribing of non-opioid analgesic pharmacotherapy, as well as clinician referrals for non-pharmacologic pain treatment (e.g., pain medicine, physical therapy, occupational therapy, rehabilitation medicine, pain psychotherapy), complementary and integrative pain therapies (e.g., acupuncture, chiropractic, massage, yoga, Tai Chi, home exercise), and specialty mental health and substance use disorder treatment. Non-opioid analgesic pharmacotherapy and referrals for nonpharmacologic pain treatments and specialty care were assessed over the year following LTOT discontinuation. Because this study aimed to characterize primary care clinicians' efforts to treat pain in patients whose opioid therapy they discontinued, we focused on clinician referrals for nonpharmacologic pain treatment and specialty care rather than actual patient engagement in these services, as patients may not participate in recommended treatment for reasons outside the control of the referring clinician.

Administrative Data Abstraction. Data abstracted from the VA Corporate Data Warehouse included sociodemographic characteristics of patient age, gender, race and ethnicity, and rurality of patients' place of residence based on rural-urban commuting area codes. ${ }^{8}$ Medical diagnoses were obtained over the 12 months prior to discontinuation of LTOT. We retrieved diagnoses of mental health disorders (depressive, bipolar, post-traumatic stress, other anxiety, and psychotic disorders), pain syndromes (musculoskeletal, neuropathic, and headache pain including migraine), and substance use disorders (alcohol, amphetamine, cannabis, cocaine, opioid, sedative, and other substance use disorders) over the 12 months prior to the discontinuation date. Additional diagnoses were used to compute a medical comorbidity score as assessed by the Elixhauser Comorbidity Index. ${ }^{9}$ Counts of pain treatment encounters - including pain medicine, rehabilitation medicine and physical therapy, occupational therapy, specialty mental health, and specialty substance use disorder treatment-were also obtained for the 12 months prior to LTOT discontinuation. Finally, we computed patients' average daily dose of opioids in milligrams of morphine equivalents for the year prior to LTOT discontinuation.

\section{Statistical Analyses}

All data were checked for completeness and accuracy prior to conducting statistical analyses. Descriptive data were summarized for sociodemographic and clinical variables. Sample characteristics were compared between patients discontinued 
from LTOT due to aberrant behaviors versus other reasons using independent samples $t$ tests for continuous variables and chi-square tests of association for categorical variables. Logistic regression models examined the association between reason for LTOT discontinuation (aberrant behavior versus other reason) and six dichotomous pain care outcomes assessed over the 12 months following discontinuation of LTOT (all coded as yes versus no): use of an opioid taper, initiating new nonopioid analgesic pharmacotherapy, referral for nonpharmacologic pain treatment, referral for complementary and integrative pain therapy, referral for specialty mental health treatment, and referral for specialty substance use disorder treatment. Adjusted models controlled for pre-specified variables found to be associated with discontinuation of opioid therapy among veterans in prior studies. ${ }^{10}$ These included age, gender, race/ethnicity, medical comorbidities, mental health diagnoses, substance use disorder diagnoses, chronic pain diagnoses, pain treatment utilization, and opioid dose prior to discontinuation. All inferential analyses utilized two-tailed tests of significance and alpha $=0.05$.

\section{RESULTS}

Similar to the population of veterans prescribed opioid therapy through VA, ${ }^{11}$ the study sample had a mean age of 55 years, was predominantly male (94\%), non-Hispanic white (71\%), and resided in urban locations (74\%). The majority of patients $(85 \%)$ were diagnosed with musculoskeletal pain, $5 \%$ had neuropathic pain diagnoses, while $10 \%$ had headache diagnoses. By study design, rates of substance use disorders were high, the most common being alcohol use disorder (diagnosed in $28 \%$ of patients) and opioid use disorder (diagnosed in $13 \%$ of patients). The most commonly diagnosed mental health disorders in the sample included post-traumatic stress disorder (31\%), other anxiety disorders (25\%), and depressive disorders (24\%). In the year prior to LTOT discontinuation, $79 \%$ of patients had also been prescribed non-opioid analgesic pharmacotherapy. Fewer patients had utilized non-pharmacologic pain treatments, including specialty pain medicine $(17 \%)$, rehabilitation medicine and physical therapy $(7 \%)$, and occupational therapy (4\%). Two thirds of patients $(67 \%)$ had at least one specialty mental health encounter in the year prior to LTOT discontinuation, while $17 \%$ of patients had at least one specialty substance use disorder treatment encounter. Seventyfive percent of patients $(n=382)$ were discontinued from LTOT by the opioid-prescribing clinician due to aberrant behaviors, while the remaining $25 \%$ of patients $(n=127)$ were discontinued for other reasons. Table 1 provides additional characteristics of the overall study sample, and the subsamples of patients discontinued from LTOT for aberrant behaviors versus other reasons.

Fifteen percent of patients in the full sample $(n=74)$ received an opioid taper and $45 \%(n=225)$ were prescribed new non-opioid analgesic pharmacotherapy or received a dose modification to one or more existing non-opioid analgesic medications. Fifty-eight percent of patients $(n=$ 296) were referred for non-pharmacologic pain treatment (including pain medicine, physical therapy, occupational therapy, rehabilitation medicine, or pain psychotherapy), while $25 \%(n=125)$ were referred for complementary and integrative pain therapies (including acupuncture, chiropractic, massage, yoga, Tai Chi, and home exercise). Referrals for specialty mental health disorder treatment were made for $65 \%$ of patients $(n=330)$, and $34 \%$ of patients $(n=175)$ received referrals for specialty substance use disorder treatment.

As shown in Table 2, in both unadjusted and adjusted logistic regression models, patients discontinued due to aberrant behaviors, relative to patients discontinued for other reasons, were more likely to receive an opioid taper (18 versus $4 \%$, adjusted $\mathrm{OR}=5.6095 \% \mathrm{CI}=2.10-14.93$ ), receive a new non-opioid analgesic medication or dose change to an existing non-opioid analgesic medication (49 versus $33 \%$, adjusted $\mathrm{OR}=2.61,95 \% \mathrm{CI}=1.59-4.29)$, and be referred for specialty substance use disorder treatment (42 versus $11 \%$, adjusted $\mathrm{OR}=7.39,95 \% \mathrm{CI}=3.76-14.53)$. Patients discontinued due to aberrant behaviors and patients discontinued for other reasons did not differ in rates of clinician referrals for nonpharmacologic pain treatment (59 versus 56\%, adjusted $\mathrm{OR}=$ $1.12,95 \% \mathrm{CI}=0.71-1.76$ ), complementary and integrative pain therapies ( 26 versus $21 \%$, adjusted $\mathrm{OR}=1.29,95 \% \mathrm{CI}=$ $0.76-2.19$ ), or specialty mental health treatment (66 versus $61 \%$, adjusted $\mathrm{OR}=1.40,95 \% \mathrm{CI}=0.85-2.30)$.

\section{DISCUSSION}

This study examined rates of new non-opioid pain treatment following discontinuation of LTOT by the opioidprescribing clinician. Three fourths of patients were discontinued due to aberrant behaviors, such as aberrant urine drug tests or concurrent substance use or alcohol use. Relative to patients discontinued from LTOT for nonaberrant reasons (e.g., safety concerns, lack of opioid treatment efficacy), those discontinued due to aberrant behaviors were more likely to be tapered from opioids, referred to specialty substance use disorder treatment, or receive some modification to the non-opioid analgesic pharmacotherapy treatment regimen (in the form of a new medication or dose change of an existing medication). These results point to the need for more intensive care when treating pain in patients discontinued from LTOT due to aberrant behaviors, particularly substance use, as active substance use may contribute to patients' levels of pain, ${ }^{12-13}$ resulting in refractory pain if the substance use disorder is not concurrently treated. Findings further highlight the need for behavioral health specialists practicing in primary care settings to be trained in evidence-based brief interventions that address both substance use disorders and chronic pain. 
Table 1 Characteristics of Patients Discontinued from Long-Term Opioid Therapy by the Opioid-Prescribing Clinician

\begin{tabular}{|c|c|c|c|c|}
\hline \multirow[t]{2}{*}{ Variable } & $\begin{array}{l}\text { Overall sample } \\
N=509\end{array}$ & $\begin{array}{l}\text { Discontinued for aberrant } \\
\text { behavior } n=382\end{array}$ & $\begin{array}{l}\text { Discontinued for other } \\
\text { reasons } n=127\end{array}$ & \multirow[t]{2}{*}{$p$ value } \\
\hline & $\%(n)$ or $\mathrm{M} \pm \mathrm{SD}$ & & & \\
\hline \multicolumn{5}{|l|}{ Sociodemographic characteristics } \\
\hline Age, in years & $55 \pm 10$ & $55 \pm 10$ & $55 \pm 13$ & 0.97 \\
\hline Male gender & $94 \%(480)$ & $96 \%(365)$ & $91 \%(115)$ & 0.04 \\
\hline Race/ethnicity & & & & 0.19 \\
\hline White, non-Hispanic & $71 \%(360)$ & $69 \%(262)$ & $77 \%(98)$ & \\
\hline Black, non-Hispanic & $17 \%(86)$ & $19 \%(72)$ & $11 \%(14)$ & \\
\hline Hispanic & $2 \%(11)$ & $2 \%(9)$ & $2 \%(2)$ & \\
\hline Other/unknown & $10 \%(52)$ & $10 \%(39)$ & $10 \%(13)$ & \\
\hline Rural-urban continuum & & & & 0.50 \\
\hline Isolated & $5 \%(25)$ & $5 \%(18)$ & $6 \%(7)$ & \\
\hline Small rural & $12 \%(62)$ & $13 \%(49)$ & $10 \%(13)$ & \\
\hline Large rural & $8 \%(43)$ & $9 \%(36)$ & $6 \%(7)$ & \\
\hline Urban & $74 \%(377)$ & $73 \%(278)$ & $78 \%(99)$ & \\
\hline Unknown & $<1 \%(2)$ & $<1 \%(1)$ & $<1 \%(1)$ & \\
\hline \multicolumn{5}{|l|}{ Clinical characteristics } \\
\hline Elixhauser Comorbidity Index & $1.6 \pm 1.4$ & $1.5 \pm 1.4$ & $1.9 \pm 1.4$ & 0.02 \\
\hline \multicolumn{5}{|l|}{ Mental health diagnoses } \\
\hline Depressive disorder & $24 \%(124)$ & $25 \%(94)$ & $24 \%(30)$ & 0.82 \\
\hline Bipolar disorder & $8 \%(41)$ & $9 \%(33)$ & $6 \%(8)$ & 0.40 \\
\hline Post-traumatic stress disorder & $31 \%(156)$ & $28 \%(106)$ & $39 \%(50)$ & 0.01 \\
\hline Other anxiety disorder & $25 \%(129)$ & $24 \%(93)$ & $28 \%(36)$ & 0.37 \\
\hline Psychotic disorder & $8 \%(41)$ & $7 \%(28)$ & $10 \%(13)$ & 0.30 \\
\hline \multicolumn{5}{|l|}{ Substance use disorder diagnoses } \\
\hline Alcohol use disorder & $28 \%(140)$ & $28 \%(108)$ & $25 \%(32)$ & 0.50 \\
\hline Amphetamine use disorder & $2 \%(11)$ & $2 \%(8)$ & $2 \%(3)$ & 0.86 \\
\hline Cannabis use disorder & $6 \%(30)$ & $7 \%(27)$ & $2 \%(3)$ & 0.05 \\
\hline Cocaine use disorder & $8 \%(40)$ & $9 \%(36)$ & $3 \%(4)$ & 0.02 \\
\hline Opioid use disorder & $13 \%(67)$ & $14 \%(55)$ & $9 \%(12)$ & 0.15 \\
\hline Sedative use disorder & $3 \%(13)$ & $3 \%(11)$ & $2 \%(2)$ & 0.42 \\
\hline Other substance use disorder & $6 \%(31)$ & $8 \%(29)$ & $2 \%(2)$ & 0.01 \\
\hline \multicolumn{5}{|l|}{ Pain diagnoses } \\
\hline Musculoskeletal & $85 \%(433)$ & $84 \%(322)$ & $87 \%(111)$ & 0.40 \\
\hline Neuropathy & $5 \%(28)$ & $5 \%(17)$ & $9 \%(11)$ & 0.07 \\
\hline Headache & $10 \%(53)$ & $10 \%(39)$ & $11 \%(14)$ & 0.80 \\
\hline Opioid dose in milligrams daily morphine equivalents & $76 \pm 90$ & $80 \pm 92$ & $65 \pm 80$ & 0.13 \\
\hline \multicolumn{5}{|l|}{ Pain treatment utilization } \\
\hline Non-opioid analgesic pharmacotherapy & $79 \%(402)$ & $78 \%(296)$ & $84 \%(106)$ & 0.15 \\
\hline Specialty pain medicine & $17 \%(86)$ & $17 \%(64)$ & $17 \%(22)$ & 0.88 \\
\hline Rehabilitation medicine/physical therapy & $7 \%(34)$ & $7 \%(25)$ & $7 \%(9)$ & 0.83 \\
\hline Occupational therapy & $4 \%(22)$ & $4 \%(14)$ & $6 \%(8)$ & 0.21 \\
\hline Specialty mental health & $67 \%(342)$ & $66 \%(253)$ & $70 \%(89)$ & 0.42 \\
\hline Specialty substance use disorder & $14 \%(69)$ & $16 \%(61)$ & $6 \%(8)$ & 0.01 \\
\hline
\end{tabular}

An encouraging finding was the rate of referral for nonpharmacologic pain treatment following opioid discontinuation, suggesting some clinicians attempted a multimodal approach to pain care. Nearly three out of five patients were referred for non-pharmacologic pain treatments, while one fourth received a referral for complementary and integrative pain therapies. That rates of referral were not higher may reflect lack of availability of some services in the years for which this study's data were collected (i.e., 2012-2013). Recent efforts to increase access to non-pharmacologic pain treatment in the VA include a national rollout of training in evidence-based cognitive behavioral therapy for chronic pain ${ }^{14}$ and the VA's Opioid Safety Initiative, ${ }^{15}$ which launched in 2013 and emphasized the use of non-opioid pharmacotherapy and nonpharmacologic pain treatments for chronic pain. In addition, some patients face access to care barriers such as geographic isolation, precluding travel to urban VA medical centers where the preponderance of specialty care is offered. ${ }^{16}$ Although rates of referral in this study may not be indicative of current practice within the VA, they provide a comparison for future studies to examine changes in referral rates for non-opioid pain treatments.

Clinical guidelines for the management of opioid therapy available at the time patients in this study were discontinued from LTOT recommended taper of opioid therapy, when appropriate, as part of the discontinuation process. ${ }^{17-18}$ In this study sample, only $15 \%$ of patients received an opioid taper. We were unable to ascertain from the electronic health record instances for which clinicians believed an opioid taper was inappropriate. In no cases did we identify clinician recommendations for medically supervised detoxification in lieu of a prescribed opioid taper. Medically supervised detoxification has been recommended as an alternative to opioid taper when an immediate discontinuation is clinically indicated, as has initiation of opioid agonist therapy for patients with opioid use disorder. ${ }^{19}$ Our findings suggest detoxification is underutilized and 
Table 2 Association of Opioid Therapy Discontinuation Reason with Pain Care Outcomes

\begin{tabular}{lll}
\hline \hline $\begin{array}{l}\text { Pain care } \\
\text { outcomes }\end{array}$ & $\begin{array}{l}\text { Unadjusted } \\
\text { odds ratio } \\
\text { (95\% confidence } \\
\text { interval) }\end{array}$ & $\begin{array}{l}\text { Adjusted odds } \\
\text { ratio (95\% } \\
\text { confidence } \\
\text { interval)* }\end{array}$ \\
\hline Opioid taper & $\begin{array}{l}5.38 \\
(2.12-13.65)\end{array}$ & $\begin{array}{l}5.60 \\
(2.10-14.93)\end{array}$ \\
$\begin{array}{l}\text { Prescribed non-opioid } \\
\text { analgesic pharmacotherapy }\end{array}$ & $1.92(1.26-2.93)$ & $2.61(1.59-4.29)$ \\
$\begin{array}{l}\text { Referral for } \\
\text { non-pharmacologic } \\
\text { pain treatment }\end{array}$ & $1.13(0.75-1.70)$ & $1.12(0.71-1.76)$ \\
$\begin{array}{l}\text { Referral for non- } \\
\text { pharmacologic } \\
\text { complementary and integrative } \\
\text { treatment }\end{array}$ & $1.28(0.79-2.07)$ & $1.29(0.76-2.19)$ \\
$\begin{array}{l}\text { Referral for specialty mental } \\
\text { health treatment }\end{array}$ & $1.22(0.80-1.85)$ & $1.40(0.85-2.30)$ \\
$\begin{array}{l}\text { Referral for specialty substance } \\
\text { use disorder treatment }\end{array}$ & $\begin{array}{l}5.88 \\
(3.26-10.62)\end{array}$ & $\begin{array}{l}7.39 \\
(3.76-14.53)\end{array}$ \\
\hline
\end{tabular}

Odds ratios greater than 1 indicate patients discontinued due to aberrant behaviors were more likely than patients discontinued for other reasons to receive an opioid taper, non-opioid analgesic prescription, pain treatment referral, or specialty mental health or substance use disorder treatment referral

*Covariate-adjusted models control for patient age, gender, racelethnicity; pre-discontinuation medical comorbidities as assessed by the Elixhauser Comorbidity Index, mental health diagnoses, substance use disorder diagnoses, and pain diagnoses; prediscontinuation opioid dose measured in milligrams of morphine equivalents; and pre-discontinuation non-opioid analgesic pharmacotherapy, pain medicine, rehabilitation medicine/physical therapy, occupational therapy, specialty mental health, and specialty substance use disorder treatment encounters

should be considered when immediate discontinuation of opioid therapy is warranted, and opioid agonist treatments are not indicated for, or declined by, the patient.

This study has several limitations. First, the study sample comprised patients with substance use disorders and matched controls and may not generalize to the greater population of VA or non-VA patients discontinued from opioid therapy by opioid-prescribing clinicians. Second, some patients may have declined clinicians' treatment referrals. We did not capture these data if they were not documented in the medical record. Third, we did not assess actual patient engagement in non-pharmacologic pain treatments and specialty care services. Although the focus of the current study was on actions taken by clinicians to treat patients' pain in the wake of LTOT discontinuation, patient engagement in recommended treatment is a necessary precursor to treatment-related improvements in patient outcomes. Future studies should examine ways to increase patient engagement in non-opioid treatment and specialty mental health and substance use disorder care following discontinuation of LTOT. Fourth, data on complementary and integrative pain treatments in the year prior to LTOT discontinuation were not collected. We were thus unable to control for pre-discontinuation utilization patterns when examining treatment utilization rates following LTOT discontinuation. Finally, data were exclusively collected through electronic health record review and administrative data abstraction. Activities that occur in pain treatment visits may not be comprehensively documented in the electronic health record (e.g., patients verbally decline treatment referrals and formal consults are never placed), ${ }^{20}$ and misclassification may thus be present.

In conclusion, overall rates of non-opioid analgesic prescribing and referral for non-pharmacologic pain treatment following clinician-initiated discontinuation of LTOT were modest, suggesting room for improvement in widening the availability of these therapies and making them more easily accessible for primary care patients. Additional improvements in opioid discontinuation processes that involve an opioid taper, medically supervised opioid detoxification, or induction of opioid agonist treatments, as indicated, may improve patients' experiences when discontinued from LTOT and increase their willingness to try non-opioid analgesic pharmacotherapy and non-pharmacologic pain treatments.

Acknowledgements: This work was supported by Locally Initiated Project Award no. QLP 59-048 (PI: Lovejoy) from the US Department of Veterans Affairs Substance Use Disorder Quality Enhancement Research Initiative. Dr. Lovejoy received additional support from Career Development Award IK2HX001516 from the US Department of Veterans Affairs Health Services Research and Development during preparation of this manuscript. We thank the VA Portland Health Care System and the US Department of Veterans Affairs Health Services Research and Development Center to Improve Veteran Involvement in Care (CIVIC; CIN 13-404, PI: Dobscha) at the VA Portland Health Care System for the provision of support and resources for this project.

Corresponding Author: Travis I. Lovejoy, PhD, MPH; Center to Improve Veteran Involvement in Care, VA Portland Health Care System, Portland, OR, USA (e-mail: travis.lovejoy@va.gov).

\section{Compliance with Ethical Standards:}

Disclaimer: The views expressed in this article are those of the authors and do not necessarily reflect the position or policy of the U.S. Department of Veterans Affairs or U.S. Government.

Conflict of Interest: The authors declare that they have no conflicts of interest.

\section{REFERENCES}

1. Pezalla EJ, Rosen D, Erensen JG, Haddox JD, Mayne TJ. Secular trends in opioid prescribing in the USA. J Pain Res. 2017;10:383-7.

2. Gellad WF, Good CB, Shulkin DJ. Addressing the opioid epidemic in the United States: lessons learned from the Department of Veterans Affairs. JAMA. 2017;177:611-2.

3. Lovejoy TI, Morasco BJ, Demidenko MI, Meath THA, Frank JW, Dobscha SK. Reasons for discontinuation of long-term opioid therapy in patients with and without substance use disorders. Pain. 2017;158:52634.

4. Dowell D, Haegerich TM, Chou R. CDC guideline for prescribing opioids for chronic pain-United States, 2016. JAMA. 2016;315:1624-45.

5. The Opioid Therapy for Chronic Pain Work Group. VA/DoD Clinical Practice Guideline for Opioid Therapy for Chronic Pain. Washington, DC: U.S. Department of Veterans Affairs/U.S. Department of Defense; 2017.

6. Morasco BJ, Duckart JP, Dobscha SK. Adherence to clinical guidelines for opioid therapy for chronic pain in patients with substance use disorder. J Gen Intern Med. 2011;26:965-71.

7. Morasco BJ, Corson K, Turk DC, Dobscha SK. Association between substance use disorder status and pain-related function following 12 months of treatment in primary care patients with musculoskeletal pain. J Pain. 2011;12:352-9. 
8. Morrill R, Cromartie J, Hart G. Metropolitan, urban, and rural commuting areas: toward a better depiction of the United States settlement system. Urban Geogr. 1999;20:727-48.

9. Elixhauser A, Steiner C, Harris DR, Coffey RM. Comorbidity measures for use with administrative data. Med Care. 1998;36:8-27.

10. Vanderlip ER, Sullivan MD, Edlund MJ, Martin BC, Jortney J, Austen M, Woilliams JS, Hudson T. National study of discontinuation of longterm opioid therapy among veterans. Pain. 2014;155:2673-9.

11. Mosher HJ, Krebs EE, Carrel M, Kaboli PJ, Weg MW, Lund BC. Trends in prevalent and incident opioid receipt: an observational study in Veterans Health Administration 2004-2012. J Gen Intern Med. 2015;30:597-604.

12. IIgen MA, Perron B, Czyk EK, McCammon RJ, Trafton J. The timing and onset of pain and substance use disorders. Am J Addict. 2010;19:409-15.

13. Morasco BJ, Lovejoy TI, Ilgen MA. Management of chronic pain in patients with a comorbid substance use disorder. In Turk DC, Gatchel RJ, eds. Psychological Approaches to Pain Management: A Practitioner's Handbook. $3^{\text {rd }}$ Ed. New York, NY: The Guilford Press; 2018.

14. Stewart MO, Karlin BE, Murphy JL, Raffa SD, Miller SA, McKellar J, Kerns RD. National dissemination of cognitive-behavioral therapy for chronic pain in veterans: therapist and patient-level outcomes. Clin J Pain. 2015;31:722-729.

15. Lin LA, Bohnert ASB, Kerns RD, Clay MA, Ganoczy D, Ilgen MA. Impact of the Opioid Safety Initiative on opioid-related prescribing in veterans. Pain. 2017;158:833-839.

16. Teich J, Ali MM, Lynch S, Mutter R. Utilization of mental health services by veterans living in rural areas. J Rural Health. 2017;33:297304.

17. Chou R, Fanciullo GP, Fine PG, et al. Clinical guidelines for the use of chronic opioid therapy in chronic noncancer pain. J Pain. 2009; 10: 113-30.

18. U.S. Department of Veterans Affairs/U.S. Department of Defense. VA/ DoD Clinical Practice Guideline for Management of Opioid Therapy for Chronic Pain. Washington, DC: Veterans Health Administration, 2010.

19. Kleber HD. Pharmacologic treatments for opioid dependence: detoxification and maintenance options. Dialogues Clin Neurosci. 2007;9:455-70.

20. Krebs EE, Ramsey DC, Miloshoff JM, Bair MJ. Primary care monitoring of long-term opioid therapy among veterans with chronic pain. Pain Med. 2011;12:740-6. 